\title{
Diffusion entropy analysis on the scaling behavior of financial markets
}

\author{
Shi-Min Cai ${ }^{1}$, Pei-Ling Zhou ${ }^{1}$, Hui-Jie Yang ${ }^{2}$, Chun-Xia Yang ${ }^{1}$, Bing-Hong Wang $^{2}$, and Tao Zhou ${ }^{1,2}$ \\ ${ }^{1}$ Department of Electronic Science and Technology, \\ University of Science and Technology of China, \\ Hefei Anhui, 230026, PR China \\ ${ }^{2}$ Department of Modern Physics, \\ University of Science and Technology of China, \\ Hefei Anhui, 230026, PR China
}

(Dated: August 6, 2018)

\begin{abstract}
In this paper the diffusion entropy technique is applied to investigate the scaling behavior of financial markets. The scaling behaviors of four representative stock markets, Dow Jones Industrial Average, Standard\&Poor 500, Heng Seng Index, and Shang Hai Stock Synthetic Index, are almost the same; with the scale-invariance exponents all in the interval [0.92, 0.95]. These results provide a strong evidence of the existence of long-rang correlation in financial time series, thus several variancebased methods are restricted for detecting the scale-invariance properties of financial markets. In addition, a parsimonious percolation model for stock markets is proposed, of which the scaling behavior agrees with the real-life markets well.
\end{abstract}

PACS numbers: 89.90.+n,05.10.-a,05.45.Tp,64.60.Cn,87.10.+e

\section{INTRODUCTION}

Analysis of financial time series attracts special attentions from diverse research fields for several decades. It can not only reveal the intrinsic dynamical properties of the corresponding financial markets but also provide us a clear scenario to construct dynamical models. Traditional theories are constructed based upon some basic hypothesis, to cite examples, the stochastic processes in the markets and the homogenous property of the markets, etc. The unexpected so-called rare events are explained simply as the results due to accidents or external triggers [1]. The advancements in nonlinear theory lead a complete revolutionary in our ideas about financial markets. Instead of the deduced Gaussian distribution, empirical investigations in recent years indicate that the price return distribution of the financial time series generally obeys the centered Lévy distribution and displays fat-tail property, and the financial time series exhibits the scale-invariance behavior [2, 3, 4, 5, 6, 7]. The nonlinear theory based analysis and dynamical models for the financial markets are the essential problems at present time.

One of the important features of the financial time series is the scale-invariance property, which can highlight the dynamical mechanics for the corresponding markets. Consider a complex system containing a large amount of particles. The scale-invariance property in the diffusion process of this system can be described mathematically with the probability distribution function as

$$
P(x, t)=\frac{1}{t^{\delta}} F\left(\frac{x}{t^{\delta}}\right),
$$

where $x$ is the displacements of the particles in the complex system and $\delta$ the scale-invariance exponent. The theoretical foundation of this property is the Central Limit Theorem and the Generalized Central Limit Theorem [8, [9]. For $\delta=0.5$, the diffusion process is the standard diffusion and $F(y)$ is the Gaussian function. And $\delta \neq 0.5$ exhibits the deviation of the dynamical process from the normal stochastic one. For a financial time series, the delay-register vectors, denoted with $\left\{y_{k}, y_{k+1}, \cdots, y_{k+m-1} \mid k=1,2,3, \cdots, N-m+1\right\}$, can be regarded as the trajectories of $N-m+1$ particles during the period of 0 to $m$. By this way we can map a time series to a diffusion process, called overlapping diffusion process in this paper. An alternative solution is to separate the considered time series into many nonoverlapping segments and regard these segments as the trajectories.

In literature, several variance-based methods are proposed to detect the scale-invariance properties, such as the probability moment method [10], the fluctuation approach and the de-trended fluctuation approach 11], etc. But these variance-based methods have two basic shortcomings. One is that the scale-invariance property can be detected but the value of the exponent cannot be obtained correctly. The other is that for some processes, like the Lévy flight, the variance tends to infinite and these methods are unavailabel at all. Although the infinite can not be reached due to the finite records of empirical data, clearly we can not obtain correct information about the dynamics under these conditions. Hence, in this paper we suggest the using of diffusion entropy (DE) technique to detect the scaling behavior of financial markets.

*Electronic address: zhutou@ustc.edu 


\section{DIFFUSION ENTROPY TECHNIQUE AND DATA ANALYSIS}

To overcome the above shortcomings in the variancebased methods, the authors in reference [12] designed the diffusion entropy analysis (DEA). To keep our description as self-contained as possible, we review the DEA method briefly.

Filter out the trends in the original time series. Adopting the traditional assumption generally used in the research filed of engineering, that a discrete time series variable consists of a slowly varying part and a fluctuation part 13,14$]$, the index of a stock market reads,

$$
\xi_{j}=S_{j}+\zeta_{j}, j=1,2, \cdots, N,
$$

where $\zeta_{j}$ is the fluctuation with zero mean and fixed variance.

In the signal processing, the slow and regular variation $S_{j}$ is usually called signal, which contains the useful information. And the rapid erratic fluctuation $\zeta_{j}$ is called the noise, which is regarded as perturbations containing only trivial information. In the DEA method, however, the scale-invariance will be detected from this fluctuation part. The "step smoothing" 13 procedure is employed to estimate the $S_{j}$ part in our calculations, that is, regard the average of the segments as the trends, respectively. The final time series is regarded as a steady series, whose overlapping diffusion process reads,

$$
x_{k}(t)=\sum_{j=k}^{k+t} \zeta_{j}, k=1,2, \cdots, N-t+1 .
$$

Consequently, the Shannon entropy can be defined as,

$$
S(t)=-\int_{-\infty}^{+\infty} P(x, t) \log _{10}[P(x, t)] d x .
$$

A simple algebraic leads to,

$$
S(t)=A+\delta \log _{10}(t)
$$

where

$$
A=-\int_{-\infty}^{+\infty} F(y) \log _{10}[F(y)] d x, y=\frac{x}{t^{\delta}} .
$$

The DEA method has been used to deal with many time series in different research fields, such as the solar induced atmosphere temperature series 15], the intermittency time series in fluid turbulence 16 , the spectra of complex networks [17, and the output spike trains of neurons 18.

In order to truly uncover the scaling behavior of financial market, we study both the domestic and oversea stock markets. The domestic consist of Shang Hai Stock Synthetic Index (SS) with length $N=5412$, and Hang Seng Index (HSI) with length $N=191076$, while Dow Jones Industrial Average (DJIA) with length



FIG. 1: (Color online) DE results for four time series from different stock markets. The squares, circles, upward triangles and downward triangles denote the results for HIS, SS, S\&P500 and DJIA, respectively. The corresponding scaling exponents are $\delta_{H S I}=0.944 \pm 0.004, \delta_{S S}=0.923 \pm 0.004$, $\delta_{S \& P 500}=0.950 \pm 0.005$ and $\delta_{D J I A}=0.948 \pm 0.005$, The solid line, whose slope is $\delta_{\text {fit }}=0.945$, is presented as reference. The occurrence of saturation regime caused by the "Step smoothing" is $t=365$. Consequently, the fitting interval should be in the range of $t<\frac{T}{3}[13]$. In the fitting procedure is implemented in the interval of $t \in[1,100]$

$N=29627$ and Standard\&Poor 500 (S\&P500) with length $N=5695$ are constituted of the oversea. In Fig. 1, the DE technique is applied to analysis these real financial time series and we demonstrate the scaling behavior of different Indices are almost same,with the scaling exponents all in the interval [0.92, 0.95]; as showing below: $\delta_{s s}=0.923 \pm 0.004, \delta_{H S I}=0.944 \pm 0.004$, $\delta_{D J I A}=0.948 \pm 0.005$, and $\delta_{S \& P 500}=0.950 \pm 0.005$. These results provide a strong evidence of the existence of long-rang correlation in financial time series, thus several variance-based methods are restricted for detecting the scale-invariance properties of financial markets.

Herein it must be noted that the occurrence of saturation regime caused by the "Step smoothing" is $t=365$. Consequently, the fitting interval must be only a limited range of times, $t<\frac{T}{3}[13$, which we estimate to be $t \in[1,100]$.

\section{THE MODEL}

There are many modelling methods to explain the origins of the observed nonlinear scaling behavior of market price as emerging from simple behavioral rules of a large number of heterogeneous market participants, such as behavior-mind models 19, 20], dynamic-games models 21, 22, cellular-automata models 23, 24], multiagent models 25, 26], and so on. Here in this paper, 
we consider a stock market model based on percolation theory 27. Cont and Bouchaud 28 successfully applied percolation theory to modelling the financial market $(\mathrm{CB}$ model), which is one of the simplest models able to account for the main stylized fact of financial markets, e.g. fat tails of the histogram of log-returns. Up to now, the percolation theory is widely used in modelling stock markets 29, 30, 31, 32].

Based on percolation theory, our model incorporates the following components different from the original $\mathrm{CB}$ model: (1) The cluster, defined as a set of interconnected investors, grows in a self-organized process. (2) The effect of "herd behavior" on the trade-volume is magnified step by step during the cluster's self-organized accumulating process rather than instantaneously formed like EZ model and its variety $[33,34,35,36]$. (3) Some encountering smaller clusters will form a bigger cluster through cooperating or one defeating the rivals. (4) An infinite cluster maybe exist without the need to tune $p$ to $p_{c}$ and its trade activity influence price fluctuation. (5)The number of investors participating in trading will vary dynamically. Now let's see the model.

\section{A. Dynamic of investor groups}

Initially, $M$ investors randomly take up the sites of an $L * L$ lattice. Then for each cluster, a strategy is given: buying, selling, or sleeping, which are denoted by $1,-1$ and 0 respectively. In reality, the circle of professionals and colleagues to whom a trader is typically connected evolves as a function of time: in some cases, traders are following strong herding behavior and the effective connectivity parameter $p$ is high; in other cases, investors are more individualistic and smaller values of $p$ seem more reasonable. In order to take the complex dynamics of interactions between traders into account, we assume that it undergoes the following evolution repeatedly:

(1)Growth: most of investors would like to imitate the strategies which have been adopted by many others, which induces "herd behavior" occurring. In this sense the herd behavior is amplified. Specially, the affection of the herd behavior will be magnified gradually with the increase of the number of investors adopting this strategy, i.e., with the growth of the clusters. During cluster's growth, a number of new investors will be attracted by it and become its members. In other words, every cluster will absorb new investors with the probability

$$
P_{d}(\tau)=P_{d}(\tau-1)+k\left(N_{T}-N_{T}(\tau-1)\right),
$$

where $k$ is a kinetic coefficient controlling the growth speed and $N_{T}$ is a threshold parameter (It has been validated that the value of the parameters $k$ and $N_{T}$ could be any value. These two parameters have no effects on the self-organization process of the clusters 37$]) . N_{T}(\tau-1)$ is the number of the agents along its boundary, defined as a set made up of agents which belong to a cluster and at least border on a site which isn't part of this cluster, at the last time step $\tau-1$. The new participating investor will take up the empty sites around the old clusters and imitate the same strategy as that of it. The probability $P_{d}$ is obviously limited to the range $[0,1]$ so that we have to impose $P_{d}=0$ and $P_{d}=1$, if the recurrence relationship Equ.7 gives values for $P_{d}<0$ or $P_{d}>1$.

(2) New cluster's birth: some investors will randomly and independently enter the market with the probability $P_{n}$. These investors don't belong to an arbitrary existing cluster and will take up the empty sites.

(3) Cooperation: encountering clusters will operate cooperation and confliction between them. When their strategies are same, they are thought to cluster together to form a new group of influence. Or there would be confliction between them. The consequence of confliction is that losers would be annexed by the winner and that a new and bigger cluster whose strategy inherent the winner's will be formed. The probability of cooperation or confliction is as follow, i.e., some a cluster will cooperate with or defeat others with the probability

$$
P_{m}(k) \sim\left|s_{\tau}^{k}\right|
$$

where $\left|s_{\tau}^{k}\right|$ is the size of $\mathrm{k}$-th cluster at time $\tau$.

(4) Metabolism: in reality, no matter how huge has the size of a group ever been it would collapse due to different influences such as government decision on war and peace. Some new clusters will come into the world in the wake of aging clusters' death. The probability with which an aging clusters will die is:

$$
P_{o}=\frac{x+y}{2 L}
$$

where $x$ and $y$ is the width of this cluster occurring on the lattice in the $x$ and $y$ direction. Equ.(4) indicates that the probability with which a cluster disbands would increase with the cluster growth. Once a spanning cluster exists, it would surely die. When a cluster disbands, all its members would leave the market and the sites where the death cluster ever occupied will be taken up by new investors with the probability $P_{n}$. Such occupied sites form a few new clusters. Every new cluster would be given a strategy randomly.

Although each cluster could trade with others at every trading step, the evolution frequency of the network topology should not be so often. Thus, we assume that the network structure of the market composed by investor groups would evolve every $N$ trading steps. With the evolution of this artificial stock market, the number of investors participating in trading isn't constant. The network will take on different structure; the affection of the herd behavior on the trade-volume is gradually magnified. Without any artificial adjustment of the connectivity probability $p$ to $p_{c}$, spanning cluster may exist, whose activity would influence the price fluctuation. 


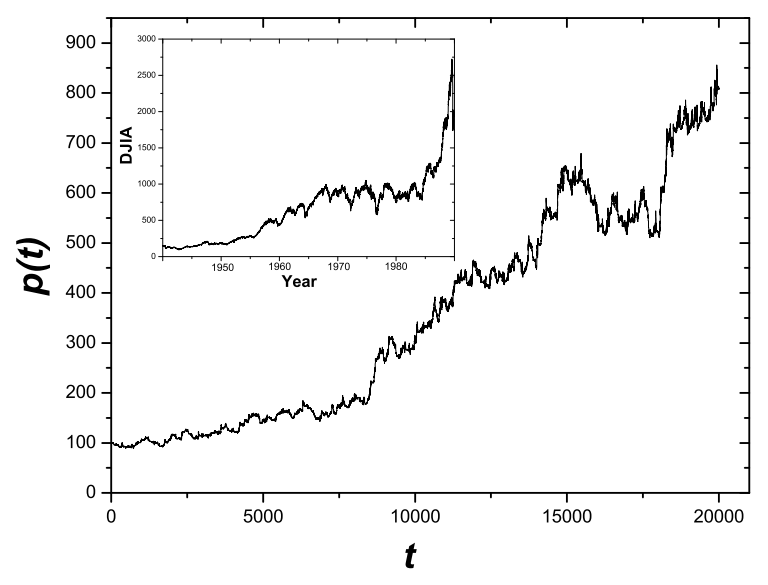

FIG. 2: (Color online) Time series of the typical evolution of the stock price in the interval. The insect is the Dow Jones Industrial Average (DJIA) from 01-02-1940 to 12-31-1987.

\section{B. Trading rules}

Each cluster trades with probability a (called activity); if it trades, it gives equal probability to a buying or selling with demand proportional to the cluster size. The excess demand is then the difference between the sum of all buying orders and selling orders received within one time interval. The price changes from one time step to the next by an amount proportional to the excess demand. To explain the "volatility", Stauffer introduces the feedback mechanism between the difference of the "supply and demand" and activity of the investors 29. Whereas in our model, the difference of the "supply and demand" not only affects the activity probability but also the probability with which the active clusters choose to buy or sell. The probability $a$ evolves following the Equ.(5):

$$
a(t)=a(t-1)+\operatorname{lr}(t-1)+\alpha,
$$

where $r$ is the difference between the demand and supply, $l$ denotes the sensitivity of $a$ to $r$ and $\alpha$ measures the degree of impact of external information on the activity. Each active cluster choose to buy or sell with probabilities $\frac{1}{2} a(t)\left(1-p_{s}(t)\right)$ and $\frac{1}{2} a(t) p_{s}(t)$ respectively. For $r>0, p_{s}(t)=0.5+d_{1} r(t-1)$, while for $r<0$, $p_{s}(t)=0.5+d_{2} r(t-1)$. According to Kahneman and Tversky [38], it is asymmetry that agents make their decisions when they are in face of gain or loss. When referring to gain, most of the agents are risk adverse. On the contrary, they are risk preference. These determine the parameters $d_{1}$ and $d_{2}$, representing the sensitivity of the agent's mentality to the price fluctuations and differing from each other. In our model we assume $d_{2}=2 d_{1}$. The difference between the demand and supply is:

$$
r(t)=\sum_{j=1}^{m} \operatorname{sign}\left(s_{t}^{j}\right)\left(\left|s_{t}^{j}\right|\right)^{\gamma}
$$



FIG. 3: (Color online) The DE result, the black squares denote the time series generated by the present model, with the scaling $\delta_{\text {model }}=0.939$, which is almost the same as that of DJIA denoted by red circles.

where $m$ is the total number of clusters occurring on the market. $\gamma$ measures the degree of impact of each cluster's trade-volume on the price, $0<\gamma<1$ allowing for a nonlinear dependence of the change of (the logarithm of the) price as a function of the difference between supply and demand [39]. So the evolution of the price is:

$$
P_{r}(t)=P_{r}(t-1) \exp (\lambda r(t))
$$

\section{Simulation}

When proper initial condition and parameters have been chosen, the artificial stock market can generate its stock price. In Fig. 2 we present a typical simulation result about price time series generated by our model, which is rather similar to the real-life index (inset). The parameters used here are $a(0)=0.09, r(0)=0.09$, $p_{r}(0)=1, P_{d}(0)=0.4, k=0.0001, N_{T}=50, l=\lambda=\frac{1}{L^{2}}$, $L=100, d_{1}=0.00005, \gamma=0.78, P_{n}=0.6, M=100$, $N=50$.

By numerical studies, we have demonstrated that this model exhibits the stylish facts with the price returns distribution is a Lévy distribution in the central part followed by an approximately exponential truncation [40], and displays power-law avalanche size distribution that agrees with the real-life markets well 41].

In succession, we investigate the scaling behavior of the stock price time series generated by the present model. One can see clearly in Fig. 3, the scaling of this artificial stock market is $\delta_{\text {model }}=0.939 \pm 0.003$, which is excellently agree with the real-life markets (see also the empirical data of DJIA for comparison). This numerical results strongly suggest that the present model has 
successfully reproduced some dynamical characters of reality.

\section{CONCLUSION}

In summary, by means of the DE method we investigate the scaling behavior embedded in the time series of four typical financial markets. The scale-invariance exponents are almost the same, being in the interval of $[0.92,0.95]$. This large deviation from the Gaussian distribution reveals the strong correlations in the time series. The present empirical study of the scaling behavior in real markets also provides a usable quantity to check the reliability of artificial market model.
Consequently, we propose a parsimonious percolation model for stock market. Proper initial condition and parameters can lead its stock price series being similar to the real-life index. Especially, the scaling behavior detected with the DE method agrees with the real-life financial markets very well.

\section{Acknowledgments}

This work has been supported by the National Science Foundation of China under Grant No. 70271070, 70471033, 10472116, and the Special Fund for the Doctoral Program of Higher Education under Grant No. 20020358009.
[1] D. Sornette, Critical Phenomena in Natural Sciences, Berlin Springer-Verlag, 2000.

[2] B. B. Mandelbrot, J. Business 36(1963) 394.

[3] R. N. Mantegna and H. E. Stanley, Nature 376(1995) 46.

[4] R. N. Mantegna and H. E. Stanley, Physica A 239(1997) 255.

[5] J. A. Lo and C. A. Mackinlay, Non-Random Walk Down Wall Street, Princeton University Press, USA, 1999

[6] B. H. Wang and P. M. Hui, Euro. Phys. J. B 20(2001) 573.

[7] H. E. Stanley, L. A. N. Amaral, X. Gabaix, P. Gopikrishnan, and V. Plerou, Physica A 299(2001) 1.

[8] S. K. Ma, Statistic Mechanics, World Scientific, Singapore, 1985.

[9] B. V. Gnedenko and A. N. Klomogorove, Limit Distributions for Sum of Independence Random Variables, Addison Wesley, Reading, 1954.

[10] G. Paladin and A. Vulpiani, Physics Reports 156(1987) 147.

[11] C. -K. Peng, S. V. Buldyrev, S. Havlin, M. Simons, H. E. Stanley, and A. L. Goldberger, Phys. Rev. E 49(1994) 1685

[12] N. Scafetta and P. Grigolini, Phys. Rev. E 66(2002) 036130 .

[13] M. Ignaccolo, P. Allegrini, P. Grigolini, P. Hamilton, and B. J. West, Physica A 336(2004) 595.

[14] M. Ignaccolo, P. Allegrini, P. Grigolini, P. Hamilton, and B. J. West, Physica A 336(2004) 623.

[15] P. Grigolini, D. Leddon, and N. Scafetta, Phys. Rev. E 65(2002) 046203.

[16] J. Bellazzini, G. Menconi, M. Ignaccolo, G. Buresti, and P. Grigolini, Phys. Rev. E 68(2002) 026126.

[17] H. -J. Yang, F. -C. Zhao, L. Qi, and B. -L. Hu, Phys. Rev. E 69 (2004) 066104.

[18] H. -J. Yang, F. -C. Zhao, W. Zhang, and Z. -N. Li, Physica A 347(2005) 704.

[19] R. Thaler, Advances in Behavioral Finances, Russell Sage Foundation, New York, 1993.

[20] A. Lo, Financial Analysis J. 55 (1999) 13.
[21] D. Frideman, Econometrica 59 (1991) 637.

[22] T. Zhou, B. -H. Wang, P. -L. Zhou, C. -X. Yang, and J. Liu, arXiv: cond-mat/0507626

[23] Y. -M. Wei, S. -J. Ying, Y. Fan and B. -H. Wang, Physica A 325(2003) 507.

[24] T. Zhou, P. -L. Zhou, B. -H. Wang, Z. -N. Tang, and J. Liu, Int. J. Mod. Phys. B 18 (2004) 2697.

[25] M. Lettau, J. Econ. Dyn. Control 21 (1997) 1117.

[26] S. -H. Chen, and C. -H. Yeh, J. Econ. Dyn. Control 25 (2001) 363.

[27] G. Grimmett, Percolation, Springer-Verlag, Berlin, 1989.

[28] R. Cont and J. P. Bouchaud, Macroeconomic Dynamics 4(2000) 170.

[29] D. Stauffer, and N. Jan, Physica A 277 (2000) 215.

[30] F. Castiglione, and D. Stauffer, Physica A 300 (2001) 531 .

[31] D. Makowiec, P. Gnaciński, and M. Miklaszewski, Physica A 331 (2004) 269

[32] J. Wang, C. -X. Yang, P. -L. Zhou, Y. -D. Jin, T. Zhou, and B. -H. Wang, Physica A 354(2005) 505.

[33] V. M. Eguíluz and M. G. Zimmermann, Phys. Rev. Lett 85(2000) 5659.

[34] D. -F. Zheng, G. J. Rodgers, P. M. Hui and R. D'Hulst, Physica A 303(2002) 176.

[35] B. Zheng, F. Ren, S. Tripmer and D. -F. Zheng, Physica A 343(2004) 653.

[36] Y. -B. Xie, B. -H. Wang, B. Hu, and T. Zhou, Phys. Rev. E 71(2005) 046135.

[37] F. S. A. Cavalcante, A. A. Moreira, U. M. S. Costa, and J. S. Andrade Jr., Physica A 311(2002) 313.

[38] D. Kahneman and A. Tversky, Econometrica 47(1979) 263.

[39] J. D. Farmer, Ind. Corp. Change 11(2002) 895.

[40] C. -X. Yang, J. Wang, T. Zhou, J. Liu, M. Xu, P. -L. Zhou, and B. -H. Wang, Chin. Sci. Bull. (In Press).

[41] P. -L. Zhou, C. -X. Yang, T. Zhou, M. Xu, J. Liu, and B. -H. Wang, New Mathematics and Natural Computation 1 (2005) 275. 